Ann. Biol. anim. Bioch. Biophys., 1979, 19 (1B), 267.275.

\title{
Prématurité de la mise bas chez la truie et signification du poids à la naissance du porcelet
}

par A. AUMAITRE, B. DEGLAIRE *, J. LEBOST

Station de Recherches sur l'Elevage des Porcs I.N.R.A. 78350 jouy-en-josas

* Union des Coopératives d'Alimentation du Bétail B. P. 75,02400 Château-Thierry.

Summary. Premature forrowing in the sow and significance of piglet birth weight.

The data on 2069 and 1881 farrowings, observed in two herds of pigs from 1972 to 1976, were used to study the effect of the length of gestation on postnatal growth and mortality of the piglets. Farrowing was experimentally induced by injection of $125 \mu \mathrm{g}$ of a prostaglandin analog (ICl 80996$)$ on day 112 (29 sows) or day 113 (20 sows) of pregnancy. We tried to characterize prematurity by the time between mating and farrowing $(\leqslant 112$ days $=$ prematurity ; 113,114, 115, 116 days = maturity $; \geqslant 116$ days $=$ post-maturity) and by mean weight classes at birth. The duration of pregnancy varied inversely with litter size at birth (fig. 2) ; less than 2 p. 100 of the animals were born before 112 days and less than 3 p. 100 after 117 days (fig. 1). Length of pregnancy did not vary with the number of the litter, but was shorter in summer than in winter. Mean piglet weight seemed to vary linearly with the length of pregnancy, i. e. from 1162 to $1334 \mathrm{~g}$ between days 111 and 118 in herd I ; however, it really varied inversely with litter size. Up to weaning at 35 days, postnatal growth rate was constant and appeared completely independent of the length of gestation. When pregnancy was experimentally shortened to 113 days, it was accompanied by a decrease in mean weight at 2 months. The number of stillborn animals was significantly higher $(P<0.01$, fig. 3$)$ when pregnancy lasted less than 112 days $(2.53$ and 1.45 per litter for herds I and II). Survival rate was minimum for animals born before 111 days (table 3). At 35 days, less than 2 animals remained out of 3 born alive in the following weight classes of herd I : less $900 \mathrm{~g}$, less than $1000 \mathrm{~g}$ for those born before 117 days, and less than $1100 \mathrm{~g}$ up to 112 days. In herd II, only animals weighing less than $900 \mathrm{~g}$ born before 117 days and those weighing less than $1000 \mathrm{~g}$ before 113 days were truly premature. Prematurity seemed to vary in swine not only with the duration of gestation and with birth weight, but also with managemental and environmental (nutrition, microclimate, quality and intensity of care at birth) conditions.

\section{Introduction.}

La durée de gestation est considérée comme peu variable dans l'espèce porcine ; aussi son influence réelle sur le poids à la naissance esł mal connue chez le porcelet pour qui l'on n'a jamais défini clairement la prématurité comme une naissance avant terme et/ou un faible poids à la naissance (Tomos, Germanova ef Izvorski, 1973 ; 
Lecyk ef al., 1976). Or on observe couramment, outre une prolificité élevée, un taux de mortalité atteignant 20 à 25 p. 100 des effectifs nés. Compte tenu du poids de la mère, celui du porcelet nouveau-né est faible : il varie de 600 à $2000 \mathrm{~g}$ avec une valeur moyenne de $1180 \pm 250 \mathrm{~g}$ dans la race Large White, et il est fortement corrélé avec le poids à deux mois $(r=+0,40)$ et le gain journalier au cours de l'allaitement (Aumaitre, Legault et Salmon-Legagneur, 1966). Le taux de survie dépend étroitement du poids à la naissance puisque $50 \mathrm{p} .100$ des porcelets nés à un poids inférieur à $900 \mathrm{~g}$ meurent dans la semaine qui suit le part (Hartsock, Graves et Baumgardt, 1977), mais peut-on considérer systématiquement ces animaux comme prématurés?

Les facteurs régulant la croissance fœetale, donc le poids à la naissance sont mieux connus : outre la race et le numéro de portée, le niveau alimentaire de la mère peut jouer un grand rôle par les apports énergétiques (Henry et Etienne, 1978) ou les apports azotés (Duée et Rérat, 1975). Mais la signification exacte du poids vif à la naissance est loin d'être établie : Curtis, Heidenreich et Martin (1967) analysant des porcelets de 420 à $1650 \mathrm{~g}$ concluent que le poids vif est sans signification sur la maturité estimée par les teneurs en constituants chimiques corporels majeurs (matière sèche, protéines, lipides). On sait que le stockage de certains constituants énergétiques importants et labiles, comme le glycogène, intervient dans les heures qui précèdent la mise bas (Salmon-Legagneur, 1968). De plus, le poids de l'embryon de porcelet qui varie de façon exponentielle avec le temps (Moustgaard, 1959) peut être affecté par la durée de gestation.

Aussi, nous avons essayé de définir la prématurité à partir d'observations sur les conséquences d'une durée de gestation variant naturellement ou expérimentalement écourtée : performances à la naissance, taux de survie et croissance des porcelets.

\section{Matériel et méthodes.}

Des enregistrements précis sur la durée de gestation ef les performances de mise bas ont été effectuées entre 1972 ef 1976 dans deux troupeaux de truies Large White. Ils concernent une première population d'animaux fécondés par des verrats de même race et ayant produit 2069 portées nées dans le troupeau de I'INRA (Elevage I); des truies fécondées par un verrat de race Landrace ayant produit 1881 portées dans le troupeau de I'UCAAB (Elevage II) complètent ces informations. Le deuxième échantillon est constitué par deux lots de truies de l'élevage l ayant subi respectivement à 112 et 113 jours de gestation une injection de $125 \gamma$ de cloprosténol (1) pour provoquer la mise bas à jour fixe (Bosc et Martinat-Botte, 1976).

Dans le but de caractériser la prématurité, nous avons classé les portées à la fois suivant la durée de gestation, distinguant des animaux normaux (nés à 113, 114, 115, 116 jours), des animaux prématurés ( $\leqslant 112$ jours) et des animaux surmaturés ( $<116$ jours), et suivant des classes arbitraires de poids moyen à la naissance définies par tranche de $100 \mathrm{~g}$ à partir de $900 \mathrm{~g}$ (tabl. 1 et 2). On a exprimé et comparé les valeurs moyennes des effectifs à la mise bas, à 35 jours, et le taux de mortinatalité

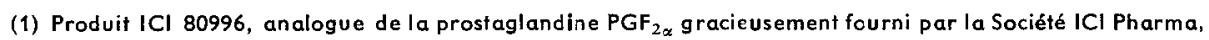
Enghien-les-Bains 95880. 
ef de mortalité postnatale. Par ailleurs, on a essayé d'estimer les conséquences de la durée de gestation sur le poids à la naissance, la vitesse de croissance et le poids à âge type des porcelets.

\section{Résultats et discussion.}

La durée de gestation varie de 108 à 119 jours dans l'élevage l ef de 101 à 120 jours dans l'élevage II ; toutefois moins de 2 p. 100 des truies ont une durée inférieure ou égale à 111 jours, et moins de 3 p. 100 une durée supérieure ou égale à 118 jours (fig. 1). Plus de 50 p. 100 des mises bas ont lieu à 114 ou 115 jours, en accord avec de nombreux auteurs, notamment Perry, 1956. Elle varie de la même façon dans les deux élevages (fig. 2) en raison inverse de la taille de la portée à la naissance, en accord avec les résultats de Lecyk et al., (1976). Un tel phénomène rencontré dans l'espèce ovine (Bosc et Cornu, 1976) semble général à toutes les espèces, de la souris (Biggers, Curnon et Finn, 1963), à la vache (Bosc, 1978). Tcutefois, la durée de gestation n'est pas liée au numéro de portée (Test 2 I non significatif à $P<0,25$ ), en accord avec Lecyk et al., 1976. La durée moyenne observée est plus courte en période estivale d'avril-mai à septembre ( $-0,5$ jour), sans doute parce que la taille moyenne de la portée est réduite à la même époque de l'année (Legault, Dagorn et Tastu, 1975).

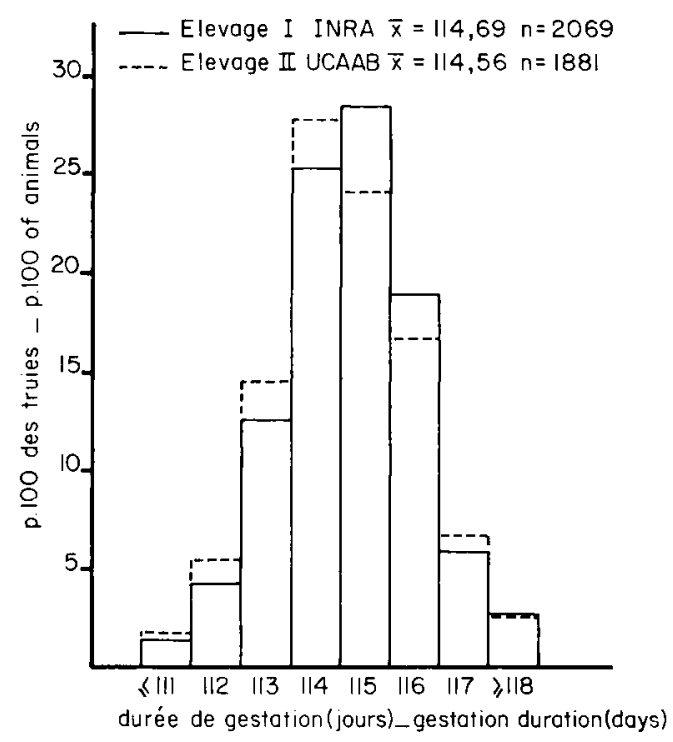

FIG. 1. - Fréquence de la durée de gestation chez la truie.

$\left(E_{\mathrm{t}} \mathrm{I}=1,48 ; \mathrm{E}_{\mathrm{t}} \mathrm{II}=1,55\right)$

Le poids moyen des porcelets nés (animaux nés totaux ou nés vivants) augmente de façon sensiblement linéaire avec la durée de gestation (fig. 2) en accord avec Tomos, Germanova ef Izvorski, 1973. Cette variation semble en contradiction avec l'évolution exponentielle du poids moyen des animaux décrite pour la période de la vie intra- 
utérine. Aussi, il nous semble difficile de définir la maturité par le seul critère du poids à la naissance, à fortiori si celui-ci est exprimé par le poids moyen de la portée.
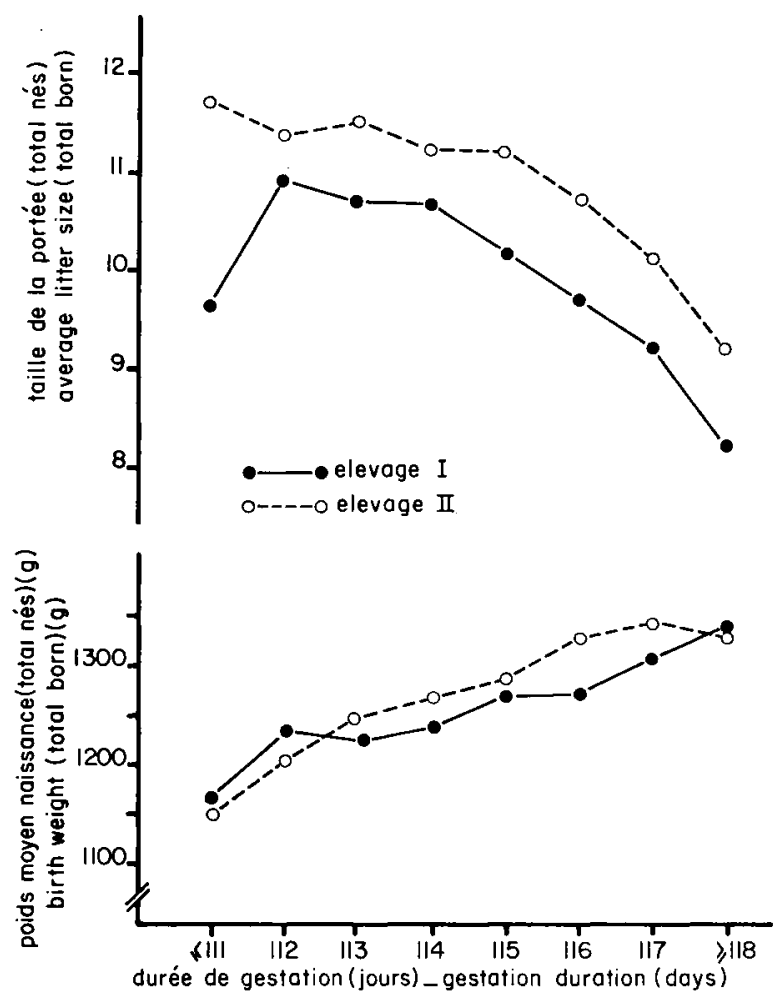

FIG. 2. - Relation entre la durée de gestation ef la taille de la portée (nombre total né) ; influence sur le poids moyen à la naissance.

On a décrit la variation du poids à la naissance suivant la durée de gestation par la fréquence en pourcentage des animaux, dans 3 classes de poids moyen : inférieur à $1000 \mathrm{~g}, 1000$ à 1200 , supérieur à $1200 \mathrm{~g}$ (łabl. 1). La fréquence maximum des porcelets de faible poids s'observe pour une durée inférieure ou égale à 112 jours (124 portées du troupeau I, 136 du troupeau II). Cette répartition différencie les deux troupeaux ur leur prolificité : la proportion d'animaux de moins de $1000 \mathrm{~g}$ est plus élevée dans le troupeau le plus prolifique (troupeau II). Chez la truie, le mécanisme hormonal de la naissance est contrôlé par un effet additif des fœtus (Bosc, du Mesnil du Buisson et Locatelli, 1974). Il est donc logique d'avoir une relation inverse entre prolificité et durée de gestation, et par conséquent les portées les plus nombreuses naissent les premières, et les porcelets ont un faible poids moyen. Toutefois l'analyse fait apparaître un pourcentage non négligeable de portées de porcelets nés largement «à terme » à un faible poids moyen. On peut donc distinguer deux types de prématurité dans l'espèce porcine, l'une liée à une courte durée de gestation, l'autre à un faible poids après une durée de gestation normale ; un phénomène identique est décrit dans d'autres espèces animales à durée de gestation beaucoup plus variable, l'homme en particulier (Davies, 1969). 
Le poids total de la portée présente un maximum aux durées de gestation les plus courtes, à partir de 112 jours dans le troupeau I et de 115 jours seulement dans le troupeau II.

\section{TABLEAU 1}

Influence de la durée de gestation sur la répartition des porcelets dans les diverses classes de poids moyen à la naissance (en p. 100 des animaux nés vivants dans chaque élevage)

\begin{tabular}{|c|c|c|c|}
\hline \multirow{2}{*}{ Durée de gestation (j) } & \multicolumn{3}{|c|}{ Classe de poids moyen ( $\mathrm{g}$ ) } \\
\hline & $\leqslant 1000$ & $1001-1200$ & $\geqslant 1201$ \\
\hline$\leqslant 112 \ldots$ & $\begin{array}{l}12,3\left({ }^{(1)}\right. \\
25,6\left({ }^{2}\right)\end{array}$ & $\begin{array}{l}31,8 \\
40,3\end{array}$ & $\begin{array}{l}55,9 \\
34,1\end{array}$ \\
\hline 113-114-115-116 $\ldots$ & $\begin{array}{r}7,0 \\
11,7\end{array}$ & $\begin{array}{l}30,7 \\
34,2\end{array}$ & $\begin{array}{l}62,3 \\
54,1\end{array}$ \\
\hline$\geqslant 117 \ldots$ & $\begin{array}{l}4,4 \\
7,0\end{array}$ & $\begin{array}{l}23,7 \\
39,6\end{array}$ & $\begin{array}{l}71,9 \\
53,4\end{array}$ \\
\hline
\end{tabular}

(1) Elevage I.

( $\left.{ }^{2}\right)$ Elevage II.

L'effectif des animaux mort-nés est significativement plus élevé $(P<0,01)$ pour les durées de gestation les plus courtes dans les deux troupeaux (fig. 3) ; il n'est pas réduit significativement par l'induction expérimentale de la parturition. Une telle situation décrite dans l'espèce ovine (Bosc et Cornu, 1976) n'a pas été trouvée par Perry (1956),

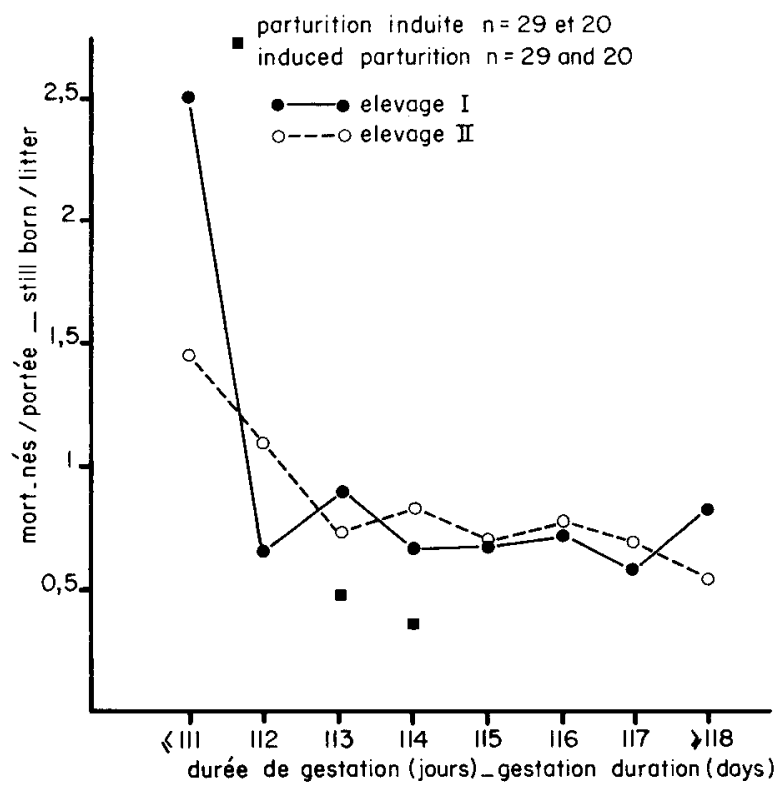

FIG. 3. - Durée de gestation et mortinatalité des porcelets. 
ni par Lecyk ef al. (1976) travaillant sur des effectifs plus limités (247 et 735 portées respectivement). Et une confusion reste toujours possible à ce niveau entre une mise bas prématurée et un avortement tardif pour des causes pathologiques.

Le taux de survie à 35 jours des porcelets nés vivants, varie aussi bien en fonction du poids moyen que de la durée de gestation. Les tableaux 2 et 3 résument pour les deux élevages l'essentiel des différences significatives observées. Ainsi les chances de survie minimum s'observent pcur les animaux nés avant 111 jours dans les 2 élevages (fig. 4). Moins de 2 animaux restent au sevrage à 35 jours sur 3 nés vivants dans l'élevage I dans les classes suivantes : inférieure à $900 \mathrm{~g}$, moins de $1000 \mathrm{~g}$ pour les porcelets nés avant 117 jours et moins de $1100 \mathrm{~g}$ pour les animaux nés jusqu'à 112 jours. Dans l'élevage II, on observe les mêmes résultats dans les seules classes: moins de $900 \mathrm{~g}$ pour les nés avant 117 jours, et moins de $1000 \mathrm{~g}$ pour les nés avant 113 jours (tabl. 2). Ce résultat nous conduit à penser que les conditions de l'élevage (soins apportés aux animaux, environnement adéquat), susceptibles de faire augmenter le taux de survie des porcelets, contribuent très largement à faire varier les conditions limites d'âge et de poids permettant de définir la prématurité.

\section{TABLEAU 2}

Taux de survie des porcelets nés vivants suivant l'élevage, la durée de gestation ef la classe de poids moyen à la naissance

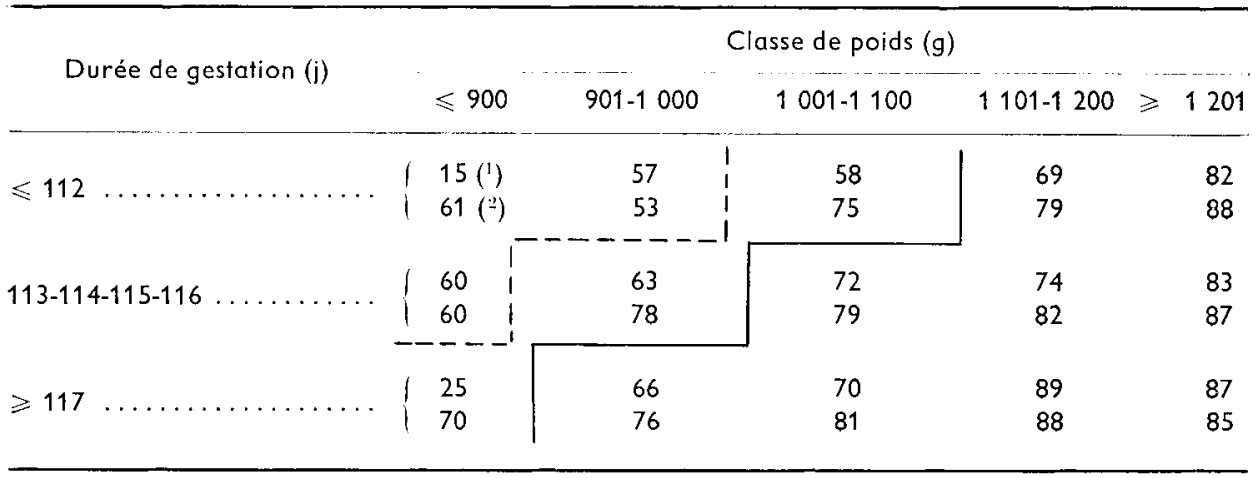

(1) Elevage I; (') Elevage II ;

$\Gamma$ et ${ }^{--}$Zone de prématurité dans les conditions de l'élevage I et II respectivement.

Une différence élevée et systématique est observée au niveau du gain de poids moyen journalier des porcelets de la naissance au sevrage entre les deux élevages (fig. 4). Celui-ci est toutefois remarquablement constant à l'intérieur d'un même troupeau quelle que soit la durée de gestation, malgré une variation de plus de $100 \mathrm{~g}$ du poids moyen à la naissance entre les gestations les plus courtes et les plus longues. Un tel résultał qui ne peut rendre compte que des performances des animaux nés vivants n'a pas été observé ef expliqué à notre connaissance. Il nous suggère que l'effet positif du poids à la naissance sur la vitesse de croissance postnatale doit se confondre le plus sou- 
vent avec les véritables facteurs de variation de cette croissance : conditions nutritionnelles (production laitière) et conditions d'environnement (microclimat, hygiène, rapidité et qualité des soins aux nouveau-nés). Ainsi la vitalité (ou le taux de survie) ne peut être définie seulement par le critère du poids moyen à la naissance.

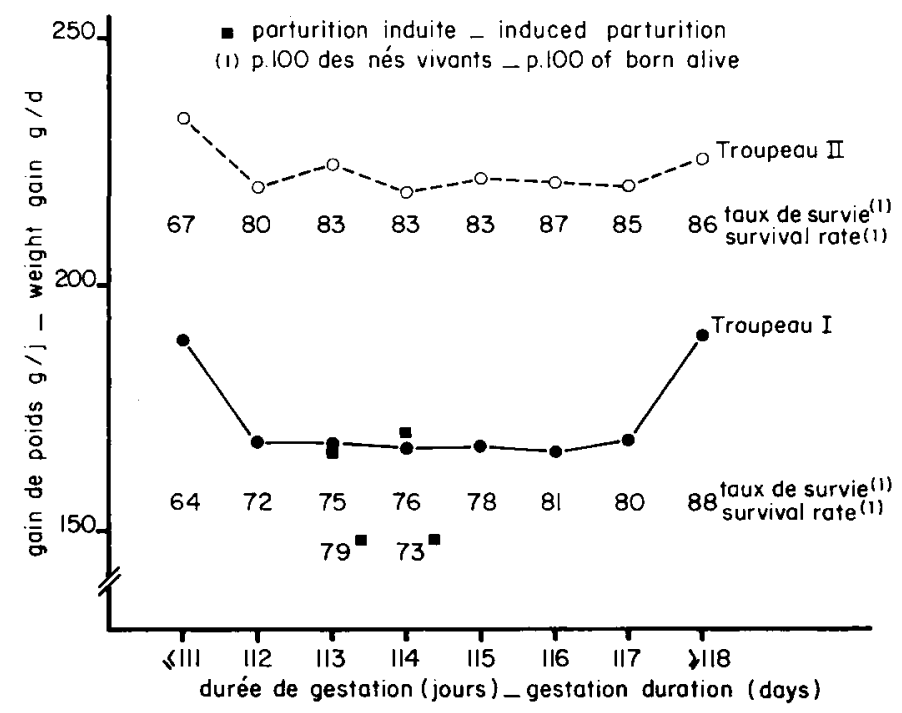

FIG. 4. - Conséquences de la durée de gestation sur le taux de survie ef la croissance postnatale.

L'induction expérimentale de la mise bas n'a pas modifié le faux de survie ni la vitesse de croissance entre la naissance et 35 jours (fig. 4), en accord avec Bosc et MartinatBotte (1976) et Elsley (1977, communication personnelle). Cependant, dans les conditions de notre élevage (tabl. 3), après avoir entraîné une baisse de poids à la naissance, elle a provoqué une diminution du poids à 2 mois dans les portées traitées à 112 jours de gestation. Des résultats voisins observés par Sugawara ef al. (1976) nous conduiraient à recommander une confirmation expérimentale de ce phénomène et une induction de parturition seulement après 112 jours de gestation (Bosc et Martinat-Botte, 1976).

TABLEAU 3

Effet de l'induction de la mise bas sur le poids à la naissance et l'évolution du poids moyen des porcelets $(\mathrm{kg})$. Elevage I

Durée de gestation (j) $113(29) \quad 114(20)$

Poids moyen à :

Naissance (nés vivants) $\ldots . \quad 1,217^{\mathrm{a}} \pm 0,170$

35 jours $\ldots \ldots \ldots \ldots \ldots \ldots \ldots \quad 7,10^{\mathrm{a}} \pm 1,84$

63 jours $\ldots \ldots \ldots \ldots \ldots \ldots \ldots \quad 15,76^{a} \pm 2,31$
$1,349 \mathrm{~b} \pm 0,256$

$7,31 \mathrm{a} \pm 1,17$

$16,59 \mathrm{~b} \pm 2,65$ 


\section{Conclusion.}

La prématurité dans l'espèce porcine n'est pas seulement liée à la mise bas avant terme. Le phénomène est accentué par l'interaction existant entre la taille de la portée et le poids moyen des porcelets. Ainsi on peut considérer comme prématurés les animaux nés à moins de $1000 \mathrm{~g}$ avant 112 jours, et les animaux nés matures en temps à moins de $1000 \mathrm{~g}$ qui n'ont pas plus de chances de survie que les animaux immatures de même poids. Les conséquences de la prématurité sur le faux de survie ef la croissance postnatale semblent dépendre des facteurs du milieu (milieu thermique, conditions nutritionnelles, prise du colostrum), et aussi des conditions d'élevage ef de l'intensité des soins.

$4^{e}$ Réunion du groupe Développement I.N.R.A. Montpellier, 17-18 mai 1978.

Remerciements. - A Michel Bosc pour ses nombreuses suggestions ef remarques sur l'intérêt ef la rédaction de ce travail.

\section{Références}

AUMAITRE A., LEGAULT C., SALMON-LEGAGNEUR E., 1966. Aspects biométriques de la croissance pondérale du porcelet. I. Influence du sexe, de l'année de naissance, du numéro et de la taille de la portée. Ann. Zootech., 15, 313-331.

BIGGERS J. D., CURNON R. N., FINN A., 1963. Regulation of the gestation period in mice. J. Reprod. Fert., 6, 125-141.

BOSC M. J., DU MESNIL DU BUISSON F., LOCATELLI A., 1974. Mise en évidence d'un contrôle fœetal de la parturition chez la truie. Interaction avec la fonction lutéale. C. R. Acad. Sci. Paris, sér. D, 278, 1507-1510.

BOSC M. J., 1978. Problèmes soulevés par la gémellité au moment de la parturition chez les bovins. Ann. Mèd. vét., 122, 5-27.

BOSC M. J., CORNU C., 1976. Ełude des facteurs affectant les conditions de mise bas et la survie des agneaux. Journ. Rech. ov. capr., INRA-ITOVIC Paris, 2, 306-321.

BOSC M. J., MARTINAT-BOTTE F., 1976. Induction de la parturition chez la truie au moyen de prostaglandines. Econ. Méd. anim., 17, 235-244.

CURTIS S. E., HEIDENREICH C. J., MARTIN T. G., 1967. Relationship between bodyweight and chemical composition of pigs at birth. J. anim. Sci., 26, 749-751.

DAVIES P. V., 1969. Feeding the newborn baby. Proc. Nutr. Soc., 28, 66-72.

DUÉE P. H., RÉRAT A., 1975. Ełude du besoin en lysine de la truie gestante nullipare. Ann. Zootech., 25, 447-464.

HARTSOCK T. G., GRAVES H. B., BAUMGARDT B. R., 1977. Agonistic behaviour and the nursing order in suckling piglets : relationships with survival growth and body composition. J. anim. Sci., 44, 320-330.

HENRY Y., ETIENNE M., 1978. Alimentation énergétique du porc. Journ. Rech. porcine en France. INRA-ITP éd., Paris, 10, 119-166.

LECYK K., NOZDRYN-PLOTNICKI J., WALKIEWICZ A., ZIN M., 1976. Effect of duration of pregnancy in Large White sows on litter size and vitality. Roczn. Nauk. Rolnic. Ser. B, 97, 103-111.

LEGAULT C., DAGORN J., TASTU D., 1975. Effet du mois de mise bas, du numéro de portée et du type génétique de la mère sur les composantes de la productivité de la truie dans les élevages français. Journ. Rech. porcine en France, INRA-ITP éd., Paris, 7, 43-51.

PERRY J. S., 1956. Observations on reproduction in a pedigree herd of Large White pigs. J. agri. Sci. Cambridge, 47, 332-443. 
MOUSTGAARD J., 1959. Nutrition and reproduction in domestic animais. In COLE D. J. A., CUPPS J., Reproduction in domestic animals, Vol. 2, 169-223. Acad. Press, New York.

SALMON-LEGAGNEUR E., 1968. Prenatal development in the pig and some other multiparous animals, 158-191. In LODGE G. A., LAMMING E., Growth and development of mammals. Butterworth, London.

SUGAWARA S., HASHIZUME K., TOTSUKAWA K., UMEZU M., MASAKI J., SATO H., ABE W., WATANABE M., USAMI N., SATO K., YOSHIDA T., 1976. Induced parfurition in swine with prostaglandin $\mathrm{F}_{2 x}$. Jap. J. Anim. Reprod., 22, 60-65.

TOMOS V., GERMANOVA L., IZVORSKI T., 1973. Abhängigkeit zwischen der Trächtigkeitsdauer beim Schwein und der Fruchtbarkeit, der Lebensfähigkeił und der Entwicklung der Ferkel während der Säugeperiode. Zhivotnovdni Nauk., 10, 115-119. 